\title{
BMJ Open Safety and immunogenicity of inactivated SARS-CoV-2 vaccines in healthy individuals: protocol for a systematic review and meta-analysis
}

\author{
Mao Li, Xuhong Yang, Lianyan Jiang (D) , Dongdong Yang (i)
}

To cite: Li M, Yang $X$, Jiang $L$, et al. Safety and immunogenicity of inactivated SARS-CoV-2 vaccines in healthy individuals: protocol for a systematic review and meta-analysis. BMJ Open 2021;11:e056106. doi:10.1136/ bmjopen-2021-056106

- Prepublication history for this paper is available online. To view these files, please visit the journal online (http://dx.doi. org/10.1136/bmjopen-2021056106).

Received 03 August 2021 Accepted 18 October 2021

Check for updates

(C) Author(s) (or their employer(s)) 2021. Re-use permitted under CC BY-NC. No commercial re-use. See rights and permissions. Published by BMJ.

Department of Neurology, Chengdu University of Traditional Chinese Medicine Affiliated Hospital, Chengdu, Sichuan, China

Correspondence to Professor Dongdong Yang; dongdongyang2020@163.com

\section{ABSTRACT}

Introduction COVID-19 has spread with high morbidity and mortality worldwide. Many inactivated SARS-CoV-2 vaccines are being tested at various clinical trial stages for the control and prevention of COVID-19. We aim to comprehensively and objectively evaluate the safety and immunogenicity of inactivated SARS-CoV-2 vaccines in healthy individuals through a systematic review and metaanalysis of randomised controlled trials (RCTs).

Methods and analysis We will search electronic databases of PubMed, the Cochrane Library, Web of Science and EMBASE for RCTs from inception to 31 December 2021. We will also search conference abstracts, reference lists, and grey literature of all available records. Two reviewers will independently screen and extract information from the literature. Bias and the quality of included studies will be evaluated with the risk-bias assessment tool provided by the Cochrane Collaboration. Statistical analysis will be performed using Cochrane's Review Manager (RevMan), V.5.3.

Ethics and dissemination Ethics approval and patient informed consent are not required because we will be including published literature only. The findings of this research will be disseminated in a peer-reviewed journal and likely through other scientific events such as conferences, seminars and symposia.

PROSPERO registration number CRD42021266285.

\section{INTRODUCTION}

The ongoing COVID-19 pandemic, caused by SARS-CoV-2, has spread worldwide causing high morbidity and mortality, and devastating the global economy. ${ }^{12}$ It has demonstrated high transmissibility in humans. According to its situation report released on 15 October 2021, the WHO estimates that COVID-19 has affected more than 240 million individuals and caused more than 4.8 million deaths worldwide. SARS-CoV-2 infects people through asymptomatic carriers and is difficult to detect, making the disease a confounding public health challenge. The clinical manifestations of COVID-19 range from asymptomatic to severe pneumonia and acute respiratory distress syndrome. ${ }^{3}$ Mild

\section{Strengths and limitations of this study}

- This systematic review will synthesise best quality evidence on the safety and immunogenicity of inactivated SARS-CoV-2 vaccines in healthy individuals from randomised controlled trials.

- This review will use a meticulous search strategy and rigorous procedure to identify and analyse all relevant peer-reviewed articles from multiple medical databases.

- Several potential sources of heterogeneity including the differences in age, regions and race may exist.

symptoms of COVID-19 that commonly occur are elevation of body temperature, coughing and breathlessness. In severe cases, organ damage can occur, leading to hospitalisation, intensive care unit admission and possibly death. ${ }^{45}$ Elderly individuals with underlying chronic conditions are at an increased risk of worse outcomes. ${ }^{6}$

From the onset of the COVID-19 pandemic, medications like antivirals, immunomodulators, corticosteroids, chloroquine and hydroxychloroquine were used for treatments. The antiviral remdesivir has shown efficacy against COVID-19 in some studies $^{78}$ and several immunomodulators have been used to reduce the generalised hyperinflammatory state caused by the cytokine storm. ${ }^{9}{ }^{10}$ However, the effects of these drugs are not desirable and their safety must be monitored carefully. ${ }^{11}$ Wearing of masks, social distancing, quarantine and isolation have played important roles in controlling the number of infected people as a short-term measure, but the absence of immunity in the population leaves them susceptible to further waves of SARS-CoV-2 infection. The lack of long-term and effective COVID-19 control has led to urgent action in the development of potential vaccines against the disease. ${ }^{12}$ Vaccination is seen as a powerful potential weapon 
in stopping the COVID-19 pandemic once herd immunity is established in the general population. Research and development (R\&D) organisations and institutes worldwide have started developing vaccines against SARSCoV-2 infection using of various vaccine platforms. Both vector-based ${ }^{1314}$ and mRNA-based vaccines ${ }^{15} 16$ have been developed and produced. For instance, vaccine named by Sputnik V, which was developed by Pfizer uses these two distinct adenovirus vectors dispensed separately in two administrations at 21 days distance from one another and induces a strong and specific antibody response against SARS-CoV-2. ${ }^{13}$ Two mRNA-based vaccines have also been trialled from safety and efficacy. ${ }^{15} 16$ These clinical studies are among the first who managed to show vaccine's safety and high efficacy in phase 3 clinical trials, to pass the official registration, and to initiate the immunisation programmes in different countries.

Considered as a more classic approach, the development of inactivated vaccines, is reliable and cost-efficient and reliable due to its long and successful history of use. ${ }^{17} 18 \mathrm{~A}$ few inactivated vaccines against COVID-19 are being developed world widely, and at least five have shown success in preclinical and clinical trials, which led to their authorisation for use. ${ }^{12}$ 19-21 Many inactivated SARS-CoV-2 vaccines are being tested at various clinical stages for the control and prevention of COVID-19. The ease of production, scale-up and relatively low cost of inactivated vaccines can enable them to capture a sizeable portion of the SARS-CoV-2 vaccine landscape. ${ }^{22}$ Inactivated vaccines can provide advantages in a variety of populations, including those with immune senescence. These vaccines are prepared based on conventional and mature vaccine production procedures. After being cultured in vitro, SARS-CoV-2 is inactivated by physical or chemical methods to ensure the nucleic acids of the virus are destroyed but remaining the integrity of its antigens. However, the 'corpse' of these viruses can still stimulate the human body to produce antibodies, inducing immune cells remember the appearance of the virus for adaptive immunity. The advantages of inactivated vaccines are as follows: (1) the production process is simple, and the technology platform for preparation and industrialisation is mature and stable. Compared with new vaccine technologies, R\&D process can start quickly; (2) the quality control points and evaluation methods are clear; (3) they have high stability; and (4) they can induce neutralising antibody responses and have good safety profiles. Based on the above advantages, an inactivated vaccine is a common means to deal with the spread of acute diseases.

The safety and efficacy of vaccines are always the concern of common people. An ideal vaccine is expected to induce protective immunity against specific pathogens without inducing any adverse reactions. However, in clinical application, the probability of adverse effects exists. ${ }^{23}$ Vaccine hesitancy is an obstacle in the establishment of herd immunity. It is defined as delayed acceptance, reluctance or refusal of vaccination even when vaccination services are available. ${ }^{24}$ The main reasons for vaccine hesitancy are doubts about the safety and efficacy of the vaccine or a belief that the virus is mild and not life-threatening. ${ }^{25}$ The accelerated speed of vaccine development has led to public anxiety and concerns regarding safety and efficacy issues, which further aggravates vaccine hesitancy. Currently, many randomised controlled trials (RCTs) evaluating the safety and immunogenicity of inactivated SARS-CoV-2 vaccines have been published. A recent systematic review was done to review the reactogenicity, immunogenicity, and efficacy of all vaccines against SARS-CoV-2, ${ }^{26}$ and another review comprehensively assessed the safety of SARS-CoV-2 vaccines. ${ }^{23}$ Another paper which systematically evaluate the effectiveness and safety of the SARS-CoV-2 vaccines currently undergoing clinical trials has also been published last month. ${ }^{27}$ In addition, the immunogenicity and safety of the CoronaVac inactivated vaccine in patients has been evaluated in patients with certain baseline conditions. ${ }^{28}$ However, there are no meta-analyses that evaluate the safety and immunogenicity of inactivated SARS-CoV-2 vaccines. Therefore, it is necessary to evaluate the safety and immunogenicity of inactivated SARS-CoV-2 vaccines through a systematic and meta-analysis of RCTs.

\section{Objectives}

The primary objective of this study is to assess the safety and immunogenicity of inactivated SARS-CoV-2 vaccines in healthy individuals through a systematic review and meta-analysis of RCTs.

\section{METHODS \\ Study guidelines and registration}

The study protocol follows guidelines of the Preferred Reporting Items for Systematic Review and Meta-Analysis Protocols. ${ }^{29}$ The systematic review and meta-analysis will be performed following the Cochrane Handbook for Systematic Reviews of Interventions and reported in compliance with the Preferred Reporting Items for Systematic Reviews and Meta-Analyses (PRISMA) statement. ${ }^{30}$ The systematic review began on 20 June 2021, and will be completed on 20 January 2022. A predetermined written protocol was registered on the PROSPERO platform (https://www.crd.york.ac.uk/PROSPERO/).

\section{Eligibility criteria}

Studies will be included in the systematic review and meta-analysis based on population, intervention, comparator, outcomes and study designs. ${ }^{19}$

\section{Population}

We will include studies that involves healthy individuals who are negative for serum-specific IgM or IgG antibodies against SARS-CoV-2 measured with a commercial kit. Demographic indicators, including age, gender and race will be not restricted in this systematic review. 


\section{Intervention}

The intervention in the experimental group is injection with inactivated SARS-CoV-2 vaccines including BBV152, CoronaVac, BBIBP-CorV, KMS-1 and WIV04 strain vaccines.

\section{Comparator}

In the control group, study participants receive a placebo using the same administration way and frequency as the experimental group. The placebo is sterile phosphatebuffered saline and alum adjuvant or aluminium hydroxide diluent solution with no virus.

\section{Outcomes}

The primary outcome will be adverse reactions, including total adverse reactions, systemic adverse reactions (fever, headache, fatigue, cough, nausea or vomiting, diarrhoea, etc) and local adverse reactions (pain, redness, swelling, itching, etc). The secondary outcome will be immunogenicity assessed as the presence of neutralising antibodies.

\section{Study types}

To maintain rigorous objectivity, this systematic review and meta-analysis will only include RCTs published in English. Quasi-RCTs, non-RCTs and any other types of studies will be excluded.

\section{Search strategy}

We will search the electronic databases from inception to 31 December 2021, for published literature on RCTs to identify eligible studies. These included PubMed, the Cochrane Library, Web of Science and EMBASE. We also searched conference abstracts, reference lists and other grey literature identified in the initial publications' search to avoid missing relevant RCTs. Missing data for studies with useful but incomplete data were obtained from the contact trial personnel for data synthesis. Previous related systematic reviews will be adopted to facilitate the searching of primary sources, but will not be included in the final results. The search strategy for PubMed is presented in table 1 .

\section{Study selection}

The reference management software EndNote X9 will be used to extract studies and remove the duplicate records using the search strategy described above. Two reviewers (XY and LJ) will independently screen literature from each electronic database by reading titles and abstracts based on the eligibility criteria. If a title or abstract is unclear, the two reviewers will review the full article. After obtaining potentially relevant studies, the reviewers will read the full-text articles for further screening and exclude those do not meet the criteria. Then, they record the reasons for each exclusion individually. If the information in the included articles is incomplete or difficult to be judged during the screening process, the reviewers will send emails to the author(s) for further information. If the original authors do not respond, the missing information will be excluded. The disagreements on study selection between the two reviewers will be resolved by discussion and arbitrated by a third reviewer (DY). Details of the entire selection procedure are shown in the PRISMA flow chart in figure 1.

\section{Data extraction}

Data extraction will be conducted using Microsoft Excel (Microsoft, Corporation, Redmond, Washington, USA). Two reviewers (XY and LJ) will independently extract the information and then will cross check the data to

Table 1 Search strategy for the PubMed

\begin{tabular}{|c|c|}
\hline Number & Search terms \\
\hline \#1 & vaccines, inactivated [Mesh] \\
\hline \#2 & $\begin{array}{l}\text { inactivated Vaccines [Title/Abstract] OR Killed vaccine [Title/Abstract] OR vaccine, killed [Title/Abstract] OR } \\
\text { vaccines, killed [Title/Abstract] OR killed vaccines [Title/Abstract] OR inactivated vaccine [Title/Abstract] OR } \\
\text { vaccine, inactivated [Title/Abstract] }\end{array}$ \\
\hline \#3 & \#1 OR \#2 \\
\hline$\# 7$ & $\begin{array}{l}\text { randomized controlled trial }[\mathrm{Pt}] \text { OR controlled clinical trial }[\mathrm{Pt}] \mathrm{OR} \text { randomized }[\mathrm{Pt}] \mathrm{OR} \text { randomly }[\mathrm{Pt}] \mathrm{OR} \\
\text { Placebo }[\mathrm{Pt}]\end{array}$ \\
\hline \#8 & \#3 AND \#6 AND \#7 \\
\hline
\end{tabular}




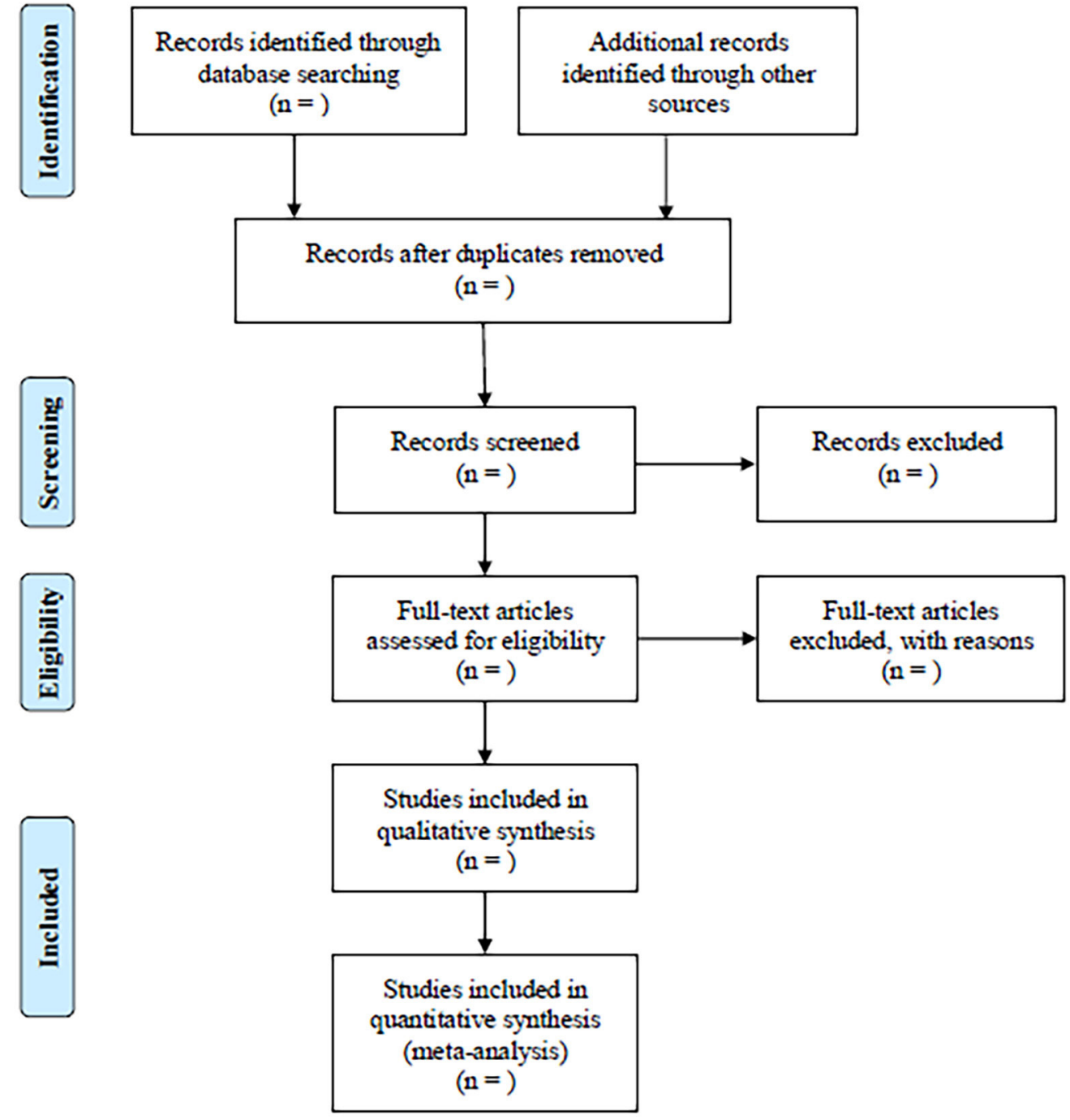

Figure 1 Flow chart of the literature search and study selection.

correct enrolment errors. The following general study information will be extracted: title, author, publication year, research country, name of the vaccine, phase 1 or 2 clinical trial, sample size, age, sex, intervention characteristics, comparator, quality evaluation method, outcomes and conclusion. Disagreements during this process will be resolved by discussing as necessary with the third reviewer (DY).

\section{Risk of bias assessment}

The two reviewers (XY and $\mathrm{LJ}$ ) will evaluate the risk of bias and the quality of included studies by using a risk-bias assessment tool provided by the Cochrane Collaboration. They will judge the risk of bias using the three categories of high risk, low risk or unclear for each item. Bias types included: (1) random sequence generation, (2) allocation hiding, (3) blinded researchers and subjects, (4) blinded comparison of the study results, (5) integrity of final data, (6) selective reporting of research results and (7) other sources. The two authors will independently review the risk of bias assessment results and the discrepancies in judgments will be resolved by consensus. If a consensus cannot be reached, the third author (DY) will be consulted.

\section{Statistical analysis}

A meta-analysis will be conducted when sufficient studies show homogeneity. Statistical analysis will be performed using Cochrane Systematic Review Manager (RevMan), V.5.3. Continuous data will be presented as the mean differences with a $95 \% \mathrm{CI}$, and dichotomous data will be presented as relative risk with a $95 \%$ CI. Statistical heterogeneity across trials will be measured by the Cochrane $Q$ test $(\mathrm{p}<0.1$ for statistical significance) and quantified by the $\mathrm{I}^{2}$ statistic. An $\mathrm{I}^{2}<50 \%$ indicates that the interstudy heterogeneity is not statistically significant, in which case the fixed-effect model will be adopted. When heterogeneity is high, we will use random-effects model without examining the probable cause for the high heterogeneity. If there is considerable heterogeneity or the data are sufficient, subgroup analysis will be conducted to identify potential sources. Subgroup analysis will be performed based on the type of adverse reactions, such as systemic adverse reactions (fever, headache, fatigue, cough, nausea 
or vomiting, and diarrhoea) and local adverse reactions (pain, redness, swelling and itching). If a study's data can not be used in our meta-analysis, we will ask the authors for relevant detailed results. If the full results can not be obtained, we will present what is provided in publications. Sensitivity analysis will be used to enhance the credibility of the results by eliminating studies with a high risk of bias, and studies with missing data and outliers if needed. Publication bias analysis will be performed using a funnel plot if more than 10 original studies included. A symmetrical funnel plot indicates a low risk of publication bias, and an asymmetrical funnel plot indicates a high risk of publication bias.

\section{Amendments}

Any future amendments to this study protocol will be summarised in the form of a table with the date of the amendment, description of deviations, and rationale provided.

Patient and public involvement we will obtain public data from published literature and authors for our systematic review. Patients or the public are not involved in the design, conduct, reporting or dissemination plans of this research.

\section{DISCUSSION}

The strengths of systematic reviews are a higher quality of evidence and better timeliness and feasibility in helping inform clinical decision-making. Many researchers have conducted clinical trials to assess the safety and efficacy of SARS-CoV-2 vaccines, but there is currently no evidencebased review that summarises the safety and immunogenicity of inactivated SARS-CoV-2 vaccines. This systematic review will synthesise and analyse the safety and immunogenicity of inactivated SARS-CoV-2 vaccines in healthy individuals. We will use a meticulous search strategy and a transparent and rigorous procedure to identify and analyse all relevant peer-reviewed articles from multiple medical databases, thereby improving the credibility of the study. With the COVID-19 pandemic continuing to threaten global public health, a vaccine is the most effective means of controlling SARS-CoV-2 infections. However, the accelerated development of vaccines has led to numerous concerns in the public about safety and efficacy issues. Our findings in this systematic review will be of great interest and value to policy makers and unvaccinated individuals especially those with vaccine hesitancy.

There are some limitations to this systematic review. First, there may be several potential sources of heterogeneity including the differences in age, regions and race. Second, the depth of this review depends on the availability of current literature. Third, although most SARSCoV-2 vaccine clinical trials are published in English, this restriction may exclude relevant data in other languages.

Contributors $X Y$ and LJ: full access to all of the data in the study and take responsibility for the integrity of the data and the accuracy of the data analysis. DY: study concept and design. ML, XY and LJ: acquisition, analysis or interpretation of data. ML: drafting of the manuscript. XY, LJ and DY: critical revision of the manuscript for important intellectual content. $X Y$ and LJ: statistical analysis. $X Y$ and LJ: administrative, technical or material support. All authors: study supervision.

Funding The authors disclosed receipt of the following financial support for the research, authorship, and/or publication of this article: This study was funded by National Key Research and Development Program of China (grant no. 2019YFC1709700 and 2019YFC1709702).

Competing interests None declared.

Patient and public involvement Patients and/or the public were not involved in the design, or conduct, or reporting, or dissemination plans of this research.

Patient consent for publication Not applicable.

Provenance and peer review Not commissioned; externally peer reviewed.

Open access This is an open access article distributed in accordance with the Creative Commons Attribution Non Commercial (CC BY-NC 4.0) license, which permits others to distribute, remix, adapt, build upon this work non-commercially, and license their derivative works on different terms, provided the original work is properly cited, appropriate credit is given, any changes made indicated, and the use is non-commercial. See: http://creativecommons.org/licenses/by-nc/4.0/.

\section{ORCID iDs}

Lianyan Jiang http://orcid.org/0000-0002-1769-7444

Dongdong Yang http://orcid.org/0000-0002-7173-5149

\section{REFERENCES}

1 Wang C, Horby PW, Hayden FG, et al. A novel coronavirus outbreak of global health concern. Lancet 2020;395:470-3.

2 Walker PGT, Whittaker C, Watson OJ, et al. The impact of COVID-19 and strategies for mitigation and suppression in low- and middleincome countries. Science 2020;369:413-22.

3 Lai C-C, Liu YH, Wang C-Y, et al. Asymptomatic carrier state, acute respiratory disease, and pneumonia due to severe acute respiratory syndrome coronavirus 2 (SARS-CoV-2): facts and myths. J Microbiol Immunol Infect 2020;53:404-12.

4 Huang C, Wang Y, Li X, et al. Clinical features of patients infected with 2019 novel coronavirus in Wuhan, China. Lancet 2020;395:497-506.

5 CDC COVID-19 Response Team. Severe Outcomes Among Patients with Coronavirus Disease 2019 (COVID-19) - United States, February 12-March 16, 2020. MMWR Morb Mortal Wkly Rep 2020;69:343-6.

6 Liu Y, Mao B, Liang S, et al. Association between age and clinical characteristics and outcomes of COVID-19. Eur Respir J 2020;55:2001112.

7 Beigel JH, Tomashek KM, Dodd LE, et al. Remdesivir for the Treatment of Covid-19 - Final Report. N Engl J Med 2020;383:1813-26.

8 Spinner CD, Gottlieb RL, Criner GJ, et al. Effect of Remdesivir vs standard care on clinical status at 11 days in patients with moderate COVID-19: a randomized clinical trial. JAMA 2020;324:1048-57.

9 Somers EC, Eschenauer GA, Troost JP, et al. Tocilizumab for treatment of mechanically ventilated patients with COVID-19. Clin Infect Dis 2021;73:e445-54.

10 Campochiaro C, Della-Torre E, Cavalli G, et al. Efficacy and safety of tocilizumab in severe COVID-19 patients: a single-centre retrospective cohort study. Eur J Intern Med 2020;76:43-9.

11 Vitiello A, Ferrara F, Porta RL. Remdesivir and COVID-19 infection, therapeutic benefits or unnecessary risks? Ir J Med Sci 2021;190:1637-8.

12 Zhang Y, Zeng G, Pan H, et al. Safety, tolerability, and immunogenicity of an inactivated SARS-CoV-2 vaccine in healthy adults aged 18-59 years: a randomised, double-blind, placebocontrolled, phase 1/2 clinical trial. Lancet Infect Dis 2021;21:181-92.

13 Logunov DY, Dolzhikova IV, Zubkova OV, et al. Safety and immunogenicity of an Rad26 and RAD5 vector-based heterologous prime-boost COVID-19 vaccine in two formulations: two open, nonrandomised phase 1/2 studies from Russia. Lancet 2020;396:887-97.

14 Voysey M, Clemens SAC, Madhi SA, et al. Safety and efficacy of the ChAdOx1 $\mathrm{nCoV}-19$ vaccine (AZD1222) against SARS-CoV-2: an interim analysis of four randomised controlled trials in Brazil, South Africa, and the UK. Lancet 2021;397:99-111.

15 Baden LR, El Sahly HM, Essink B, et al. Efficacy and safety of the mRNA-1273 SARS-CoV-2 vaccine. N Engl J Med 2021;384:403-16.

16 Polack FP, Thomas SJ, Kitchin N, et al. Safety and efficacy of the BNT162b2 mRNA Covid-19 vaccine. N Engl J Med 2020;383:2603-15. 
17 Bandyopadhyay AS, Garon J, Seib K, et al. Polio vaccination: past, present and future. Future Microbiol 2015;10:791-808.

18 Demicheli V, Jefferson T, Ferroni E, et al. Vaccines for preventing influenza in healthy adults. Cochrane Database Syst Rev 2018;2:CD001269.

19 Che Y, Liu X, Pu Y, et al. Randomized, double-blinded and placebocontrolled phase II trial of an inactivated SARS-CoV-2 vaccine in healthy adults. Clin Infect Dis 2020. doi:10.1093/cid/ciaa1703. [Epub ahead of print: 09 Nov 2020].

20 Ella R, Reddy S, Jogdand $\mathrm{H}$, et al. Safety and immunogenicity of an inactivated SARS-CoV-2 vaccine, BBV152: interim results from a double-blind, randomised, multicentre, phase 2 trial, and 3-month follow-up of a double-blind, randomised phase 1 trial. Lancet Infect Dis 2021;21:950-61.

$21 \mathrm{Wu} Z, \mathrm{Hu}$ Y, Xu M, et al. Safety, tolerability, and immunogenicity of an inactivated SARS-CoV-2 vaccine (CoronaVac) in healthy adults aged 60 years and older: a randomised, double-blind, placebo-controlled, phase 1/2 clinical trial. Lancet Infect Dis 2021;21:803-12.

22 Iversen PL, Bavari S. Inactivated COVID-19 vaccines to make a global impact. Lancet Infect Dis 2021;21:746-8.

23 Chen M, Yuan Y, Zhou Y, et al. Safety of SARS-CoV-2 vaccines: a systematic review and meta-analysis of randomized controlled trials. Infect Dis Poverty 2021;10:94.
24 MacDonald NE. Vaccine hesitancy: definition, scope and determinants. Vaccine 2015;33:4161-4.

25 Dror AA, Eisenbach N, Taiber S, et al. Vaccine hesitancy: the next challenge in the fight against COVID-19. Eur J Epidemiol 2020;35:775-9.

26 McDonald I, Murray SM, Reynolds CJ, et al. Comparative systematic review and meta-analysis of reactogenicity, immunogenicity and efficacy of vaccines against SARS-CoV-2. NPJ Vaccines 2021;6:74.

27 Ling Y, Zhong J, Luo J. Safety and effectiveness of SARS-CoV-2 vaccines: a systematic review and meta-analysis. J Med Virol 2021.

28 Medeiros-Ribeiro AC, Aikawa NE, Saad CGS, et al. Immunogenicity and safety of the CoronaVac inactivated vaccine in patients with autoimmune rheumatic diseases: a phase 4 trial. Nat Med 2021;27:1744-51.

29 Moher D, Shamseer L, Clarke M, et al. Preferred reporting items for systematic review and meta-analysis protocols (PRISMA-P) 2015 statement. Syst Rev 2015;4:1.

30 Liberati A, Altman DG, Tetzlaff J, et al. The PRISMA statement for reporting systematic reviews and meta-analyses of studies that evaluate health care interventions: explanation and elaboration. $J$ Clin Epidemiol 2009;62:e1-34. 